\title{
A Software Metric for the Activeness of a Local Area Network
}

\author{
Nidhi Garg \\ Assistant Professor \\ Department of Computer Science and Engg. \\ Manav Rachna International University \\ Faridabad, Haryana, India
}

\author{
Sachin Lakra \\ Associate Professor \\ Department of Information Technology \\ Manav Rachna University \\ Faridabad, Haryana, India
}

\begin{abstract}
The ongoing expansion in the Internet is the cause of continuous utilization and traffic behaviour changes. Due to diversity and fast changing properties, the Internet is a moving target. The tremendous increase in usage of the internet has necessitated the development of a tool that can model the suitability and quality of a network in terms of software metric. The effective management of any network requires quantification, measuring and modeling that can depict the nature of a network. This paper illustrates an approach to calculate the Activeness of a Local Area Network (LAN) and describes a tool called LAN Activeness Calculator (LANAC). The paper describes the Network Activeness Metric (NAM) which can gauge the degree of readiness of a LAN to give a response.
\end{abstract}

\section{General Terms}

Software Metric, Networking, Local Area Networks, Quality of Service (QoS)

\section{Keywords}

Local Area Network, Software Metrics, Activeness, Quality of Services, Network Activeness Metric, Local Area Network Activeness Calculator.

\section{INTRODUCTION}

Today, the Internet has emerged as the key component for commercial and personal communication. One contributing factor to the ongoing expansion of the Internet is its versatility and flexibility. Today networks have become an inseparable part of daily life. There is a need to measure and analyse network performance. For this purpose, a number of network tools have been designed to quantify different aspects of performance. As the most widely used type of network is a local area network, more and more tools are being developed for analyzing them. In this paper, an attempt has been made to design an interface for a meaningful software metric pertaining to gauging the performance of a local area network. The focus was mainly on using the quality of service parameters of a network.

The paper sets out to provide a better understanding by presenting the Internet's QoS parameters from a new point of view. The datasets that are presented are practical and taken through real-time experimentation, thus contributing to the current global understanding of the commonly used layout of networking - Local Area Networks (LANs). The primary objectives were:

- To identify the parameters that directly affect the quality of service delivered by a LAN,

- To define the term 'Activeness' with respect to networks,
- $\quad$ To find the relationship among QoS parameters so as to develop a model for calculating the activeness of a local area network,

- To derive software metric that gives a measure of a local area network's activeness.

- To develop a software tool that can calculate and display the numeric value of the activeness of a local area network.

An attempt has been made to devise a meaningful software metric by carrying out extensive research on the available literature [1-4] pertaining to local area networks and activeness, and collecting actual data from various networks. The activeness metric was then calculated using the collected data. This metric was then analysed with different values and then the actual performance of a network was obtained.

The paper is organized as follows. Section 1 introduces the problem and its background. Section 2 presents an overview of software metrics, existing software metrics and Quality of Service $(\mathrm{QoS})$ parameters. Activeness is defined with respect to local area networks in section 3. Section 4 describes the relationship between each QoS parameter and activeness, the proposed network activeness metric and the algorithm used to gauge it. Section 5 explains the tools developed and reports the sampling design used to test the derived metric. Section 6 discusses the experimental results. Section 7 highlights the merits and demerits of using the metric developed. Finally, section 8 concludes the paper with directions for future work.

\section{SOFTWARE METRICS AND NETWORK PERFORMANCE}

\subsection{Software Metrics}

Software Metrics can be defined as "The continuous application of measurement-based techniques to the software development process and its products to supply meaningful and timely management information, together with the use of those techniques to improve that process and its products"[5]. Software Metrics can provide the information needed by engineers for making technical decisions as well as quality related decisions [6]

Software Metrics are an integral part of the state-of-thepractice in software engineering. These metrics can be classified into three categories: product metrics, process metrics and project metrics. Software quality metrics are a subset of software metrics that focus on the quality aspects of the product, process and project. The essence of software quality engineering is to investigate the relationships among in-process metrics, project characteristics, and end-product quality, and based on the findings, to engineer improvements in both process and product quality. A metric quantifies 
characteristics of a process or a product. It is desirable to keep track of how well a network is working compared to its planned working. This is because the response of the network is to be found out to see whether the network is responding to requests as per given requirements or not.

The broad class of Software Metrics, into which the calculated metric falls is known as "Quality Metrics" [1]. Software quality can be depicted using various characteristics like correctness, efficiency, portability, maintainability, reliability, etc. Similarly, metrics which can gauge a network's quality based on a network's parameters are bandwidth, throughput, packet loss, delay and jitter.

\subsection{Network Performance}

The performance of networks can be measured in two broad categories, first, network efficiency, and second, network activeness. The category of efficiency involves time and space complexities. But when the discussion is regarding Networking, the time complexity gets mapped into QoS parameters such as delay or packet loss, and, the space complexity is rather ignored as the resources are shared.

There are various factors that influence the smooth working of a network. These factors are latency, packet loss, throughput, error-rate, jitter, speed, reliability, echo and bandwidth.

Some of these factors which fall under the category of Quality of Service are: [7]

1) Bandwidth: Bandwidth can be defined as the amount of data that can flow through a network over a given period of time. The bandwidth of a composite signal is the difference between the highest and lowest frequencies contained in the signal.

Bandwidth is a term used to describe the capacity of a link. It is the transmission rate for the link. Even though bandwidth is what is provided by the internet service provider, it is the throughput of the connection that is of interest for the customer. "Throughput is a measure of the amount of data that can be sent over a link in a given amount of time [8]".

The bandwidth can be formulated as:

Bandwidth $=$ Highest Frequency - Lowest Frequency (Eq. 2.1)

The throughput is determined by the formula:

$$
\begin{aligned}
\text { Throughput } & =\frac{\text { Data Transferred }}{\text { Time }} \\
& =\frac{\text { TCP Window Size }}{\text { Round Trip Time }}
\end{aligned}
$$

The throughput is expressed in bits per second or packets per second. TCP Window Size may differ from one network to another. But when expressed in bits per second, the more typical expression is kilobits (103 bits), megabits (106 bits) or gigabits (109 bits) per second, depending on the connection throughput. The difference between throughput and bandwidth is that throughput measurements may be affected by considerable overhead that is not included in bandwidth measurements. And therefore throughput is a more realistic estimator of the actual performance for the connection [8]. The two factors affecting the throughput are the amount of data transferred, and the time it took to transfer that data. Determining the throughput can be done in two ways:
Measuring the time it takes to transfer a predetermined amount of data.

Measuring the amount of data transferred in a predetermined amount of time.

2) Delay: Packet delay is the delay experienced by the packets when passing through the network. It can either be defined in terms of one-way end-to-end delay or in a round-trip manner, i.e., from sender to receiver and then from receiver to sender.

Delay $=$ Round Trip Time $/ 2$

3) Jitter: Jitter, also called delay variation, is the difference between the on-the-way delays of the selected packets. It is specified as the absolute value of delay between selected packets. The jitter between the ith and the $(\mathrm{i}-1)^{\text {th }}$ packet is given by $\mathrm{j}_{\mathrm{i}}$.

The general formula for jitter can be stated as:

$j_{i}=D_{i}-D_{i-1}$

where, $D_{i}$ is delay experienced by $i^{\text {th }}$ packet while travelling from source to destination.

4) Packet Loss: When the communication takes place through a communication link, there may be some packets that might not reach the destination. These can be calculated as the difference between the number of packets received and the total number of packets transferred.

No. of Packet Lost $=($ No. of Packets sent - No. of Packets received)

Or, expressed as a percentage,

Packet Loss $=\frac{\text { Packets sent }- \text { Packet received }}{\text { Packets Sent }} * 100$

(Eq. 2.5)

\section{ACTIVENESS}

Lakra [1] was the first to coin the term Activeness. Lakra starts by making an observation that if a human being tries to ward off a honeybee by waving a hand at it, the honeybee may get angered and may bite the human being. This implies that the honeybee is able to respond since it has the capability to do so. This capability arises from the property of the honeybee to be organized into a system which imparts to it the property of activeness, that is, the degree of readiness to respond to stimuli. His paper describes the origin of activeness and its significance in software requirements engineering.

Activeness is a term used to define the degree of readiness of a system to respond to stimuli from the environment in which it exists [1]. A system is said to have activeness because a system can be defined as a set of components that are working together to achieve a common goal [1]

Lakra et al [1] have described the importance of software metrics in and before the various phases of software engineering. Lakra et al [2] analyzed the Activeness of an Object Oriented Component Library (OOCL) and developed a special type of software metric called Component Activeness Quotient (CAQ). Lakra et al [3] proposed a software metric called Class Activeness Metric which determined the level of accessibility of the members of a class when instantiated as objects. Lakra et al [4] shed light on a Metric for the Activeness of a Distributed Object-Oriented Component Library. 


\section{THE PROPOSED METRIC}

There are a number of factors that play a vital role in measuring a network's performance. These factors can be of two types, viz., tangible or non-tangible. The tangible factors can be controlled via various means, whereas non-tangible factors are the factors that are beyond human control, i.e., they might be minimized but cannot be fully avoided. Therefore, the performance of a network can be controlled up to some order but not in totality. The tangible factors are to be looked after prior to the setup of the network, but to improve the performance of an already set-up design, one has to keep a check on non-tangible factors.

The proposed method for measuring the network performance is by checking its activeness. The term 'Activeness' with respect to computer networks can be defined as the degree of readiness of a system (which can either be a server or a client or an entire LAN) to respond to the stimuli from the environment in which it exists.

With regards to the communication over a network, the major requirement of any user is to receive a quick response. The user may desire to access a file on a remote system over a network, or he might want to use a printer or similar kind of device attached to the network. In today's jet-speed era, everything is moving within instants; of course, the user will want to find out the value of a reliability factor for each and every thing he pays for. Therefore, when a user is paying for a network, he would like to find out whether he can trust the fault free working of the system or not. Here arises the requirement of such a tool which can help users to find out the desired value, because it is known that the network performance is unreliable and time-dependent.

'The Software metric for the Activeness of a LAN' exploits the quality of service parameters of a network and binds them together to convey the degree of readiness of a network to give a response. Some parameters like throughput are directly proportional to activeness whereas there are some that are indirectly proportional.

There are various factors that affect the response of a network. The response to the demand of a file placed on a remote system that is connected to the network can be calculated using the factors that describe quality of service. By reading all the factors that affect the activeness of a network the objective of the paper was to find out the level of QoS of a LAN, by developing an Activeness Metric.
Steps followed to derive the mathematical model are as follows:

1. Find the factors that are used to calculate the QoS parameters.

2. Find out the relationship between each of these parameters and activeness.

3. Derive the mathematical model using these factors for the activeness of either a client or a server.

There are a number of factors that might affect a network's performance, but there are four major factors that were considered in this paper. These are:

1. Throughput

2. Delay

3. Jitter

4. Packet Loss

The relationship of these parameters with activeness is discussed below.

A LAN is said to be more active if it gives fast responses, and high throughput implies that a large number of packets can be transmitted in a unit amount of time.

Hence, Activeness AC of a LAN is directly proportional to throughput, that is,

$$
A_{C} \propto \text { Throughput }
$$

If the time taken by packets to travel between a sender and a receiver in a network is large and similarly, there is variation in this delay between all the packets being delivered, the LAN will give a slow response, causing it to have low activeness. Therefore, delay and jitter are inversely proportional to the activeness of a LAN.

$$
\begin{aligned}
& A_{C} \propto \frac{1}{\text { Jitter }} \\
& A_{C} \propto \frac{1}{\text { Delay }}
\end{aligned}
$$

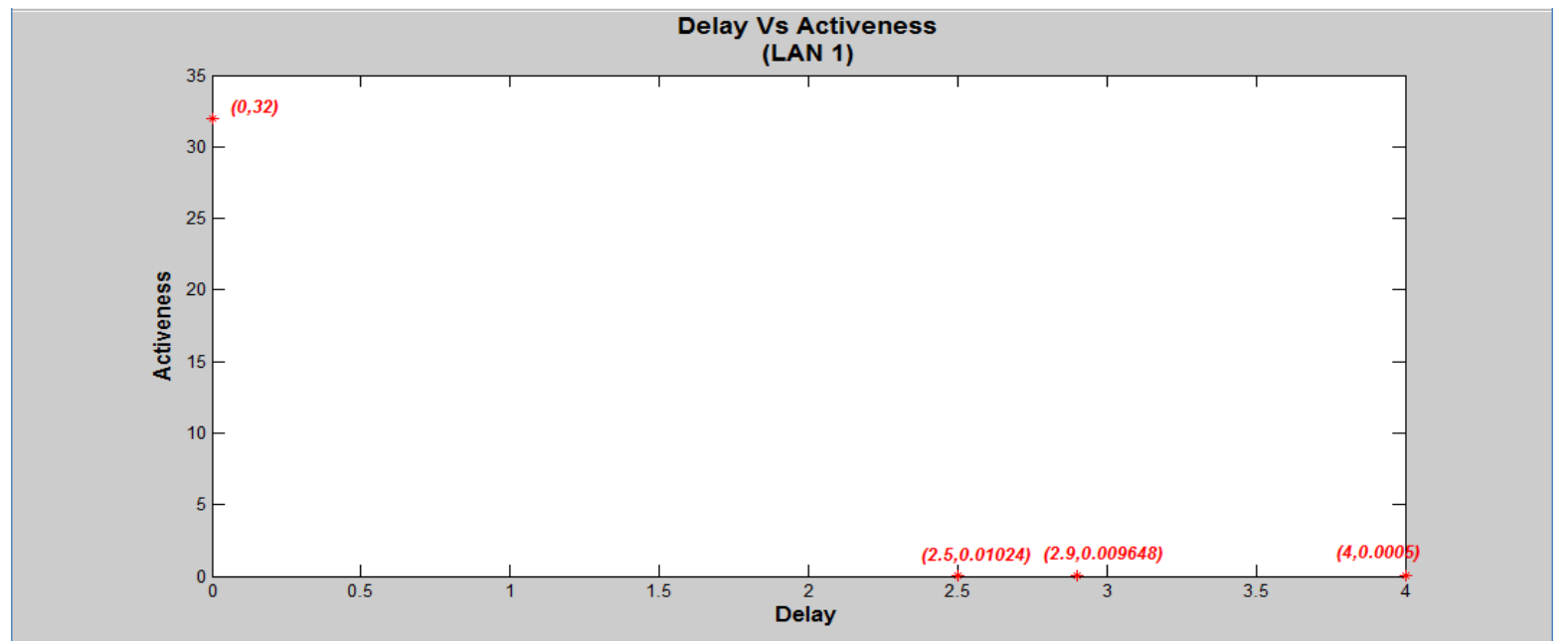

Figure 4.1: Graph for Delay vs. Activeness of LAN1 


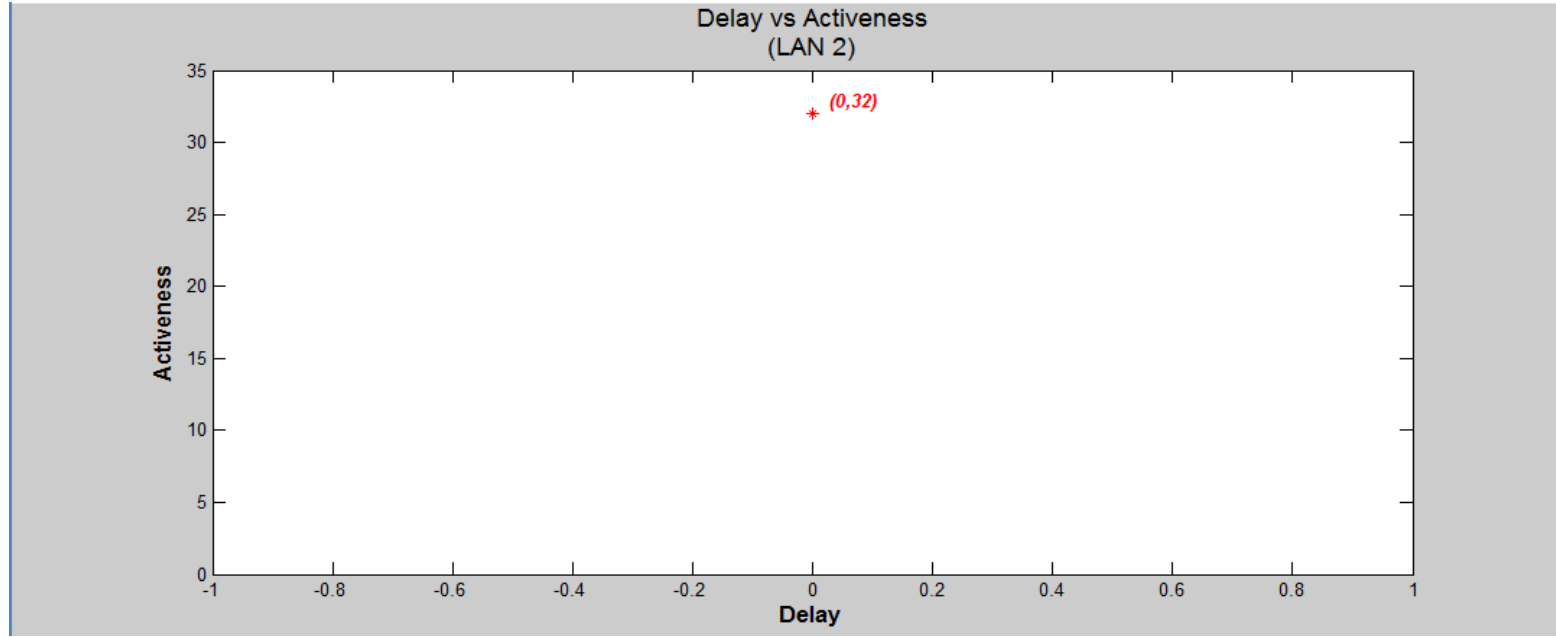

Figure 4.2: Graph for Delay vs. Activeness of LAN2

Similarly, if a large number of packets are lost during transmission, the LAN will have low activeness since the quality of the response will be degraded, leading to an inverse effect on the activeness of the LAN. Thus,

$A_{C} \propto \frac{1}{\text { PacketLoss }}$

(Eq.4.4)
From the relationships shown above, it is clear that the only parameter that is directly proportional to the activeness of a single client in a network is throughput, which is given by Eq. 2.2. The other three parameters, i.e., delay, jitter and packet loss, each show an inverse effect on the activeness of a single client.

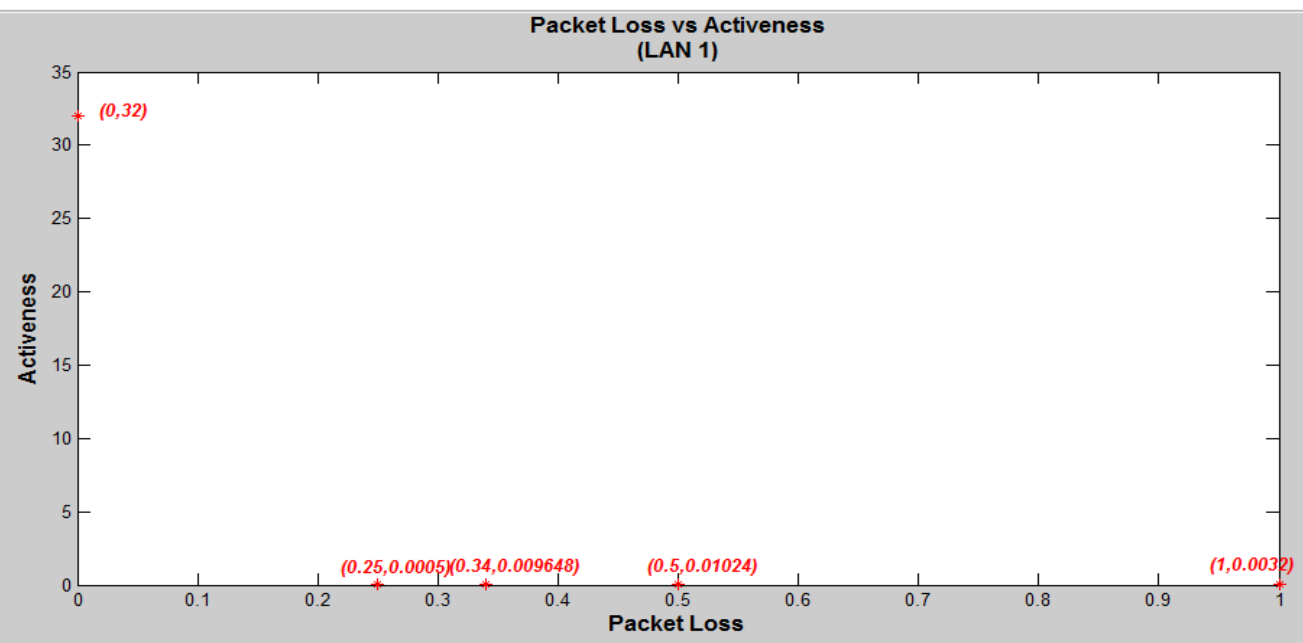

Figure 4.5: Graph for Packet Loss vs. Activeness of LAN1

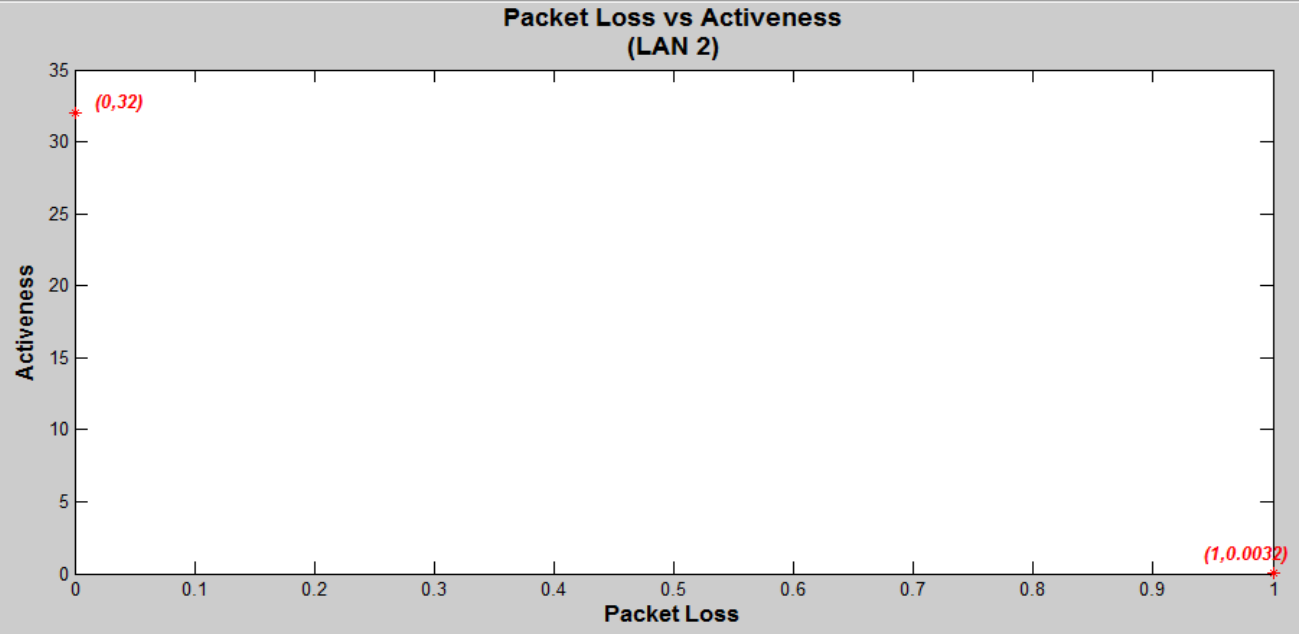

Figure 4.6: Graph for Packet Loss vs. Activeness of LAN2 
Using Eq. 4.1-4.4 and combining all the four parameters, the following mathematical model of the Activeness of a LAN is obtained:

$$
A_{C} \propto \frac{\text { Throughput }}{\text { Packet Loss } \times \text { Delay } \times \text { Jitter }}
$$

Replacing each of the parameters in Eq. 4.5 with their mathematical expressions from Eq. 2.2-2.5, we have the following mathematical model of the Activeness of a LAN:

$$
A_{C} \propto \frac{T C P \text { Windowize }}{R T T} \times \frac{2}{R T T} \times \frac{1}{\left(D_{i}-D_{i-1}\right)} \times \frac{100}{P L}
$$

This model can be used in any kind of network; therefore, the metric has been christened as the Network Activeness Metric (NAM), but the experimentation was carried out only on Local Area Networks.

\section{EXPERIMENTS AND LANAC TOOL}

The Local Area Network Activeness Calculator (LANAC) is the tool that was developed in the implementation stage and it gauges the activeness of computers organized in a clientserver architecture based network.

The tool was developed using MATLAB and contains two modules, one to calculate the activeness of a client or a standalone PC, and the second, for a server.

There are numerous network monitoring tools available for free online that enable users to analyze performance issues, raise alerts against threats, and provide reports on the health of a network's environment.

Ping is the most frequently used utility that is used by network users and the command used in our case for gauging the QoS parameters.

Another tool used to gather data is PingTester, which is a free network performance tool available online. It is a visual network test tool, and can store a list of IP addresses, URL's, and network test commands to increase the working efficiency. PingTester can perform verification with a single click.

The sample design for the research work is shown in Table 5.1 .

Table 5.1 Sample Design

\begin{tabular}{|c|c|c|c|}
\hline Network & $\begin{array}{c}\text { Number of } \\
\text { computers }\end{array}$ & $\begin{array}{c}\text { No. of } \\
\text { clients }\end{array}$ & $\begin{array}{c}\text { No. of } \\
\text { servers }\end{array}$ \\
\hline LAN 1 & 5 & 4 & 1 \\
\hline LAN 2 & 5 & 4 & 1 \\
\hline
\end{tabular}

Data for the four parameters, viz., throughput, packet loss, delay and jitter, was collected from two LANs, each with a specified number of computers, as mentioned in Table 5.1.

\subsection{LANAC Client Tool}

In the LANAC client module, any individual client connected over a network to a server will have to use the basic network command called ping to test the communication with the server. This will generate an output containing all the required information like round trip time, number of packets sent, number of packets received, the IP address of the system being pinged, etc. This output is redirected to a text file which is imported into MS Excel. The MS Excel file is then used as the input file by the tool. Another way to calculate the activeness is the manual method, in which the user has to manually enter the values of the parameters along with the IP address, and LANAC will gauge the activeness and display it. LANAC can make the exact calculation about the activeness of a LAN. The assumption made while designing this client side tool is that the system is connected to a working server.

\subsubsection{Steps to extract output from Ping Command} (in Windows $O S$ )

1. Click the start button and select Run.

2. Enter cmd in Open field and click OK.

3. Type the simple command ping followed by the IP address of the system of which the activeness is to be calculated. The command should be typed as under:

a) ping [IP-address] > [output file-name.txt]: When using a single redirect command, it will always over-write (delete) the contents of the existing file (unless one specifies a different file name) before writing any new data.

b) ping [IP-address] > [output file-name.txt]: If you want to append (concatenate) data to an existing file.

\subsubsection{Steps to import text file into Microsoft Excel}

1. Open the Microsoft Excel Workbook

2. Go to Data Tab in the menu bar and select the option 'From Text' as shown in Fig. 5.1. It will ask for the path of the text file. Select the file to be imported.

3. This will direct to the 'Text Import Wizard'. The text file can be imported in three easy steps and at last the file should be saved in .xls or .csv format.

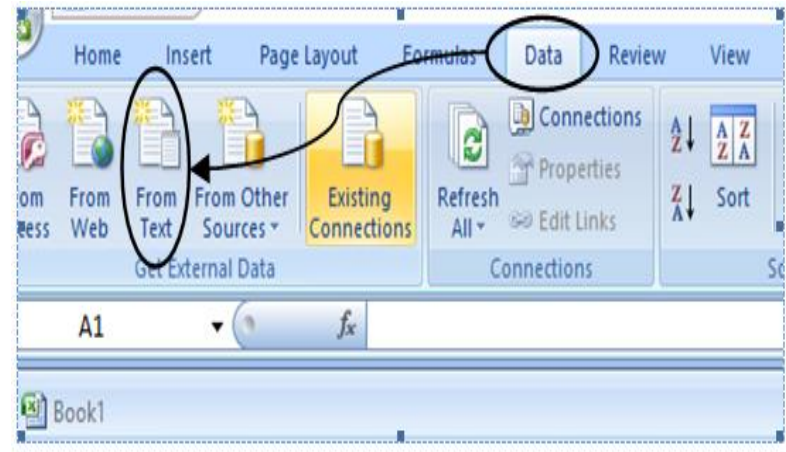

Fig. 5.1 Importing Text File

\subsubsection{Steps to run LANAC Client Tool}

The LANAC Client Tool, as shown in Fig 5.2, can be simply started by double clicking on its executable application file. There are two options provided to the tool users, which are described below:

\section{OPTION 1: Manual Approach}

This is the option selected by default. In this case, the data entry of all four parameters, viz., throughput, packet loss, delay and jitter, is to be done by the user manually. The IP address of the system being pinged is also to be specified by the user (which is not mandatory). This makes the tool difficult to use as data is to be specified statically.

The steps to be followed are: 
1. Enter the IP address (not mandatory).

2. Enter the values of all four parameters.

3. Click on 'Calculate' button. The Activeness will be calculated.
OPTION 2: Automated Approach

This option makes the tool vibrant and interesting as the data input into the fields of parameters are obtained from the file that already contains the data. When the user chooses the second option, all the edit text boxes are deactivated.

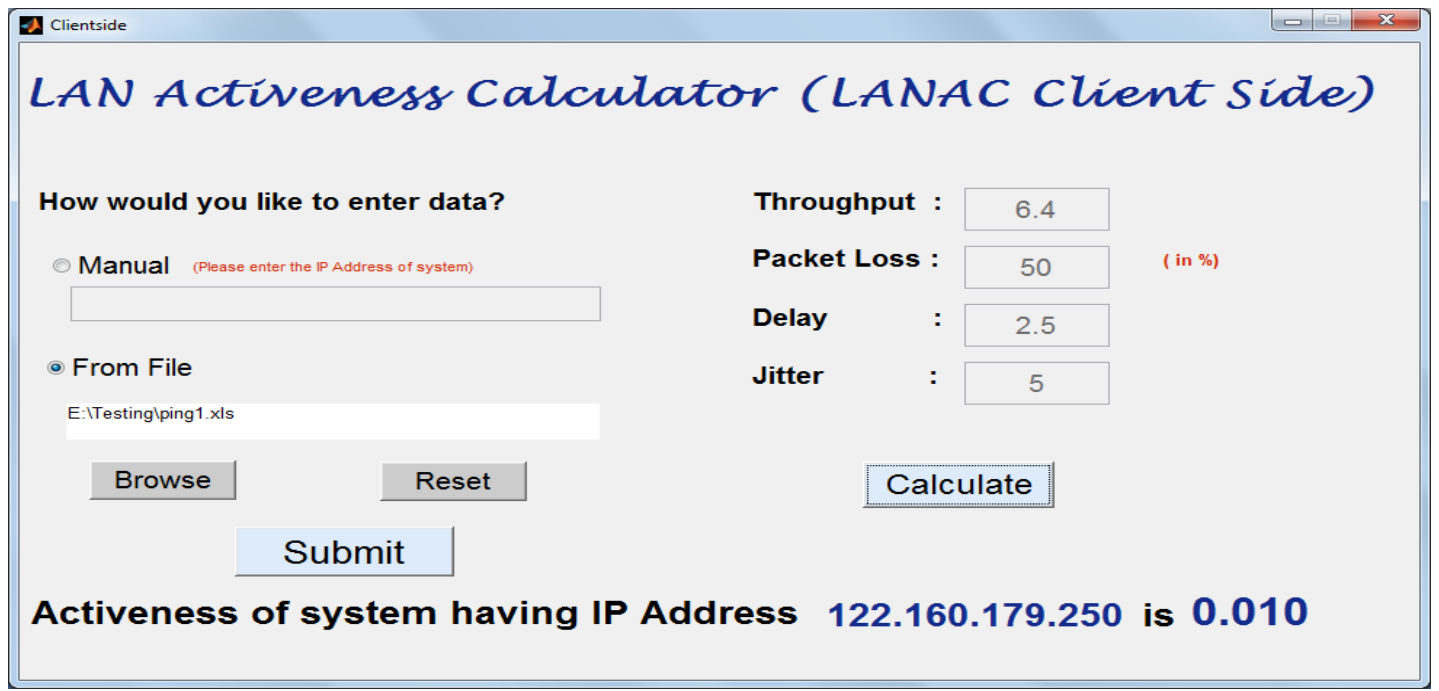

Figure 5.2: LANAC Client Side Tool

The steps to be followed are:

1. Once 'From File' is selected all the text fields except the browsing text box become inactive.

2. Click on the 'Browse' button to specify the path and select the required Excel file.

3. The Excel file is read and the values of each parameter is extracted from the file by the tool, thus making it convenient for the user.

4. Once the values are displayed in their respective text boxes, click on the 'Calculate' button to find out the activeness of the system being pinged.

5. The tool will automatically extract the IP address from the file and display it along with the activeness value obtained.

There are four push buttons provided in the tool, namely:

1. Browse Button: provides the functionality of browsing for the path of file needed.

2. Submit Button: accepts the file and gives the value of the parameters in their respective fields.

3. Calculate Button: calculates the activeness of the system, considering all four parameters and uses the mathematical model developed in this paper.

4. Reset Button: is meant to refresh the window and bring it back to initial state.

\subsection{Lanac Server Side Tool}

In the LANAC Server module, a network administrator or a user having administrator privileges can find out the activeness of the network he is connected to. The assumption made while designing the server side tool is that all the systems connected together in a network are switched on and are in a running state.

\subsubsection{Overview of PingTester Networking Tool}

One has to use a tool called PingTester that will enable the user to ping a list of systems connected over the network.

This will generate an output containing all the required information like round trip time, number of packets sent, number of packets received, and the IP addresses of all the systems being pinged. This tool allows the user to directly store the output in .xls or .csv file using the 'Export' option.

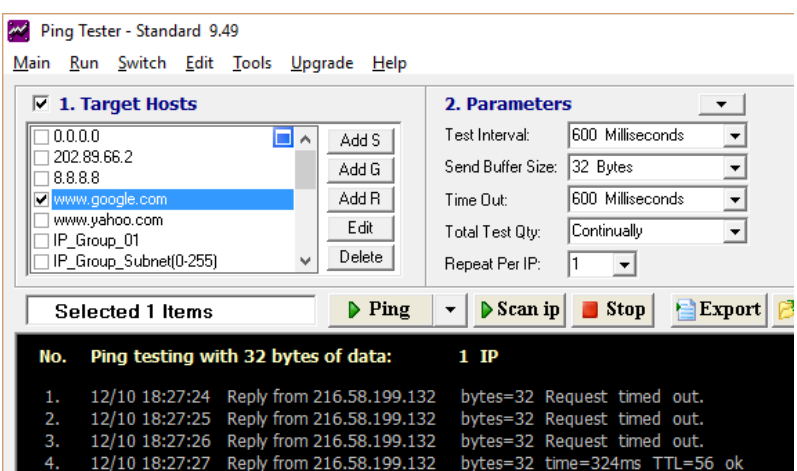

Figure 5.3: Ping Tester tool

This tool also enables the user to specify the buffer size and the number of pings to be done.

\subsubsection{Steps to run LANAC Server Tool}

The LANAC Server Tool then scans the whole file and extracts the required values. The values of parameters of all the systems are stored as an array. Thereafter the mean of each array is calculated and the value is displayed in respective fields. The LANAC Server Tool calculates the activeness of a LAN using the mathematical model discussed in Section 4. The steps to run the tool are:

1. Run the LANAC Server Tool and click on 'Browse' button to select the file.

2. Click on 'Submit' button to obtain values of throughput, packet loss, delay and jitter. 
3. Click on 'Calculate' to plot the graph and gauge the activeness of the LAN.
The graph is plotted between throughput represented on the horizontal axis and Activeness on the vertical axis.

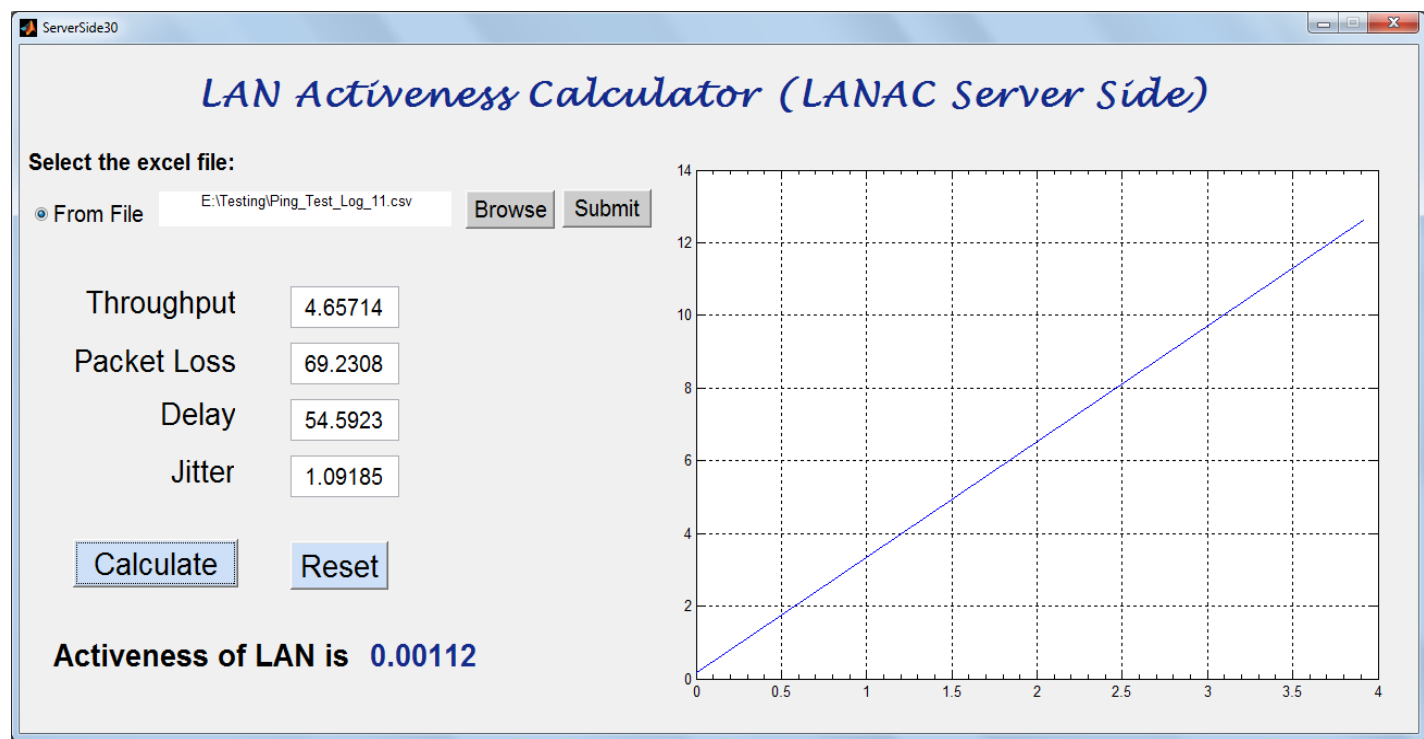

Figure 5.4: LANAC Server Side Tool

\section{RESULTS}

According to the sample design drafted, the tool was tested on two LANs each having five systems, out of which four systems were considered as clients and one system, was treated as a server

Table 6.1 presents the results of calculating the activeness of LAN 1. There were four clients, viz., C11, C12, C13 and C14, and a server named $\mathrm{S} 11$. The table was formulated by pinging the clients from server S11. The default TCP Window size of packets was 32 bytes for all the systems.
The client $\mathrm{C} 11$ reports $8 \mathrm{~ms}$ as round trip time. There was $25 \%$ of packet loss reported, i.e., there were 25 out of 100 packets that got lost during transmission. The values of the parameters delay and jitter were reported as $4 \mathrm{~ms}$ and $8 \mathrm{~ms}$, respectively. Using the mathematical model derived during the research work carried out, the activeness of the $\mathrm{C} 11$ client was obtained to be equal to 0.005 , which implies that the system was not having a high degree of response

Table 6.1 Results for LAN1

\begin{tabular}{|c|c|c|c|c|c|c|c|}
\hline \multicolumn{8}{|c|}{ LAN 1: } \\
\hline \multirow{2}{*}{$\begin{array}{c}\text { Computer } \\
\text { No. }\end{array}$} & \multirow[t]{2}{*}{ IP Address } & \multicolumn{2}{|c|}{ Throughput } & \multirow{2}{*}{$\begin{array}{c}\text { Packet Loss } \\
(\%)\end{array}$} & \multirow[t]{2}{*}{ Delay } & \multirow[t]{2}{*}{ Jitter } & \multirow[t]{2}{*}{ Activeness } \\
\hline & & $\begin{array}{l}\text { TCP Window } \\
\text { Size }\end{array}$ & $\begin{array}{c}\text { Round Trip Time } \\
\text { (RTT) }\end{array}$ & & & & \\
\hline C11. & 172.16 .16 .1 & 32 & 8 & 25 & 4 & 8 & 0.0005 \\
\hline C12. & 122.160 .179 .250 & 32 & 8 & 50 & 2.5 & 5 & 0.01024 \\
\hline C13. & 172.16 .123 .12 & 32 & 5.8 & 34 & 2.9 & 5.8 & 0.009648 \\
\hline C14. & 172.32 .11 .1 & 32 & - & 100 & - & - & 0.0032 \\
\hline S11. & 127.0 .0 .1 & 32 & 0 & 0 & 0 & 0 & 32 \\
\hline
\end{tabular}

In a similar manner, the other four systems were tested, the server S11 reported the highest activeness as there was no packet loss, zero delay and zero jitter.

Similarly, Table 6.2 depicts the results obtained for LAN 2 In this LAN all clients except $\mathrm{C} 24$ reported the least activeness, and all other clients, i.e., C21, C22, C23 and server S21 can be said to be highly active. This was since packet loss, delay and jitter for clients C21-C23 were negligible. 
Table 6.2 Results for LAN2

\begin{tabular}{|c|c|c|c|c|c|c|c|}
\hline \multicolumn{8}{|c|}{ LAN 2: } \\
\hline \multirow{2}{*}{$\begin{array}{c}\text { Computer } \\
\text { No. }\end{array}$} & \multirow[t]{2}{*}{ IP Address } & \multicolumn{2}{|c|}{ Throughput } & \multirow{2}{*}{$\begin{array}{c}\text { Packet Loss } \\
(\%)\end{array}$} & \multirow[t]{2}{*}{ Delay } & \multirow[t]{2}{*}{ Jitter } & \multirow[t]{2}{*}{ Activeness } \\
\hline & & $\begin{array}{l}\text { TCP Window } \\
\text { Size }\end{array}$ & $\begin{array}{c}\text { Round Trip Time } \\
\text { (RTT) }\end{array}$ & & & & \\
\hline C21. & 172.16 .26 .240 & 32 & 0 & 0 & 0 & 0 & 32 \\
\hline C22. & 122.160 .31 .37 & 32 & 0 & 0 & 0 & 0 & 32 \\
\hline C23. & 172.16 .27 .44 & 32 & 0 & 0 & 0 & 0 & 32 \\
\hline C24. & 172.16 .27 .45 & 32 & - & 100 & - & - & 0.0032 \\
\hline S21. & 127.16.27.47 & 32 & 0 & 0 & 0 & 0 & 32 \\
\hline
\end{tabular}

\section{MERITS AND ANALYSIS}

LANAC is characterized as one of the efficient tools to further improve the performance of networks, but the non-functional nodes in a LAN can degrade overall activeness. Most of the approaches attempt to calculate the efficiency in terms of time and space. The degree of response given by a network to a stimulus in a particular environment can be improved by integrating all four factors, namely, throughput, delay, jitter, and packet loss. The Ping Tester has been used as a tool for extracting the values of QoS parameters.

The results of this research work allow a network administrator to use the LANAC tool to accurately determine the activeness of a local area network. Awareness of activeness is necessary for a network user as it imbibes reliability in his mind.

\section{CONCLUSIONS AND FUTURE SCOPE}

This paper considers a network as a system which responds to stimuli, and gauges the activeness factor in the networking field. By carrying out research on activeness, new results have appeared for measuring the performance of a network. It lays emphasis on the parameters that determine the quality of service of a given network. The networking command ping and network measuring tool PingTester are used for measuring these parameters. Depending upon the values of these parameters, the activeness quotient is gauged for a client or a server, as required.

While carrying out the research work, it was found that, the requirement varies according to the environment and need of the user. The various parameters discussed in this paper have different importance for different types of users. For instance, a military officer may need real-time response of the request he has made, hence, the parameters that matter for him the most are minimal delay and jitter. On the other hand, a daily internet user who merely intends to send an email to his friend has least interest in the parameters like jitter and delay, but he desires that the mail he has sent should be delivered, no matter how long it takes, which implies, he requires that packet loss does not occur. These varying situations leads to the idea, whereby weights may be assigned to each of the concerned parameters and, the activeness of the network may then be calculated.

\section{REFERENCES}

[1] Sachin Lakra, Bharti Jha, Nitin Bhardwaj, Ritu Saluja and Nand Kumar: " Metrics For The Pre-Development Phase Of Software Requirements Engineering"; Proceedings (Abstract) of National Conference on Emerging Trends in Software Engineering and Information Technology, Gwalior, M.P., India; (29-30 March, 2007), 21

[2] Sachin Lakra, Nand Kumar, Sugandha Hooda, Nitin Bhardwaj, "A Metric For The Activeness Of An ObjectOriented Component Library", Proceedings of Software Engineering Research \& Practice (SERP'07), WORLDCOMP '07 - The 2007 World Congress in Computer Science, Computer Engineering, and Applied Computing, Las Vegas, Nevada, USA; $25^{\text {th }}-28^{\text {th }}$ June 2007; pp. 704-709.

[3] Sachin Lakra, Deepak Kumar Sharma, Jugnesh Kumar, Rakesh Chandra Verma and T. V. Prasad, "A Software Metric for the Activeness of a Class", International Journal of Information Technology \& Knowledge Management (Impact Factor: 0.48), Vol II, Issue I, 109113, June 2009.

[4] Sachin Lakra, T.V. Prasad, Shree Harsh Atrey and Deepak Kumar Sharma; "A Metric for the Activeness of a Distributed Object Oriented Component Library"; MR International Journal of Engineering \& Technology, Vol. 2, No.1, pp. 19-26; June, 2010.

[5] Paul Goodman, "Practical Implementation of Software Metrics”, McGraw Hill, London, UK, 1993.

[6] Westfall Linda, "12 Steps to Useful Software Metrics", The Westfall Team, Whitepaper, USA, Accessed 1 July $2007 . \quad$ Available at http://www.westfallteam.com/Papers/12_steps_paper.pdf

[7] Xiaoyuan Ta, "A Quality of Service Monitoring System for Service Level Agreement Verification", [Dissertation], School of Electrical and Information Engineering, University of Sydney, Sydney, Australia, March 2006.

[8] Joseph D. Sloan, "Network Troubleshooting Tools", O'Reilly Media Inc, California, USA, 2001. 